\title{
Autosomal dominant epilepsy with auditory features: a new LGII family including a phenocopy with cortical dysplasia
}

\author{
Karl Martin Klein ${ }^{1,2} \cdot$ Manuela Pendziwiat $^{3} \cdot$ Rony Cohen $^{4} \cdot$ Silke Appenzeller $^{3,5}$ • \\ Carolien G. F. de Kovel ${ }^{6}$ - Felix Rosenow ${ }^{1,2}$ • Bobby P. C. Koeleman ${ }^{6}$. \\ Gregor Kuhlenbäumer $^{7} \cdot$ Liron Sheintuch $^{8} \cdot$ Ronel Veksler $^{8} \cdot$ Alon Friedman $^{8}$. \\ Zaid Afawi ${ }^{9}$ - Ingo Helbig ${ }^{3,8,10}$
}

Received: 24 July 2015/Revised: 23 September 2015/Accepted: 23 September 2015/Published online: 12 October 2015 (C) Springer-Verlag Berlin Heidelberg 2015

\begin{abstract}
We report a new family with autosomal dominant epilepsy with auditory features (ADEAF) including focal cortical dysplasia (FCD) in the proband. We aim to identify the molecular cause in this family and clarify the relationship between FCD and ADEAF. A large Iranian Jewish family including 14 individuals with epileptic seizures was phenotyped including high-resolution 3-T MRI. We performed linkage analysis and exome sequencing. LGII, KANKI and RELN were Sanger sequenced. Seizure semiology of 11 individuals was consistent with ADEAF. The proband underwent surgery for right mesiotemporal FCD. 3-T MRIs in four individuals were unremarkable. Linkage analysis revealed peaks on chromosome 9p24 (LOD 2.43) and 10q22-25 (LOD 2.04). A novel heterozygous LGII mutation was identified in all affected individuals except for the proband indicating a phenocopy. Exome sequencing did not reveal variants within the
\end{abstract}

Electronic supplementary material The online version of this article (doi:10.1007/s00415-015-7921-2) contains supplementary material, which is available to authorized users.

Karl Martin Klein

k.klein@med.uni-frankfurt.de

1 Department of Neurology, Epilepsy Center Frankfurt RhineMain, Center of Neurology and Neurosurgery, University Hospital, Goethe-University Frankfurt, Frankfurt, Germany

2 Department of Neurology, Epilepsy Center Hessen, University Hospitals Giessen and Marburg, Philipps-University Marburg, Marburg, Germany

3 Department of Neuropediatrics, Christian-AlbrechtsUniversity of Kiel and University Medical Center Schleswig-Holstein, Kiel, Germany

4 Schneider Children's Medical Center of Israel, Petach Tikva, Israel and Sackler Faculty of Medicine, Tel Aviv University, Tel Aviv, Israel chromosome 9p24 region. Closely located variants in $K A N K 1$ and a RELN variant did not segregate with the phenotype. We provide detailed description of the phenotypic spectrum within a large ADEAF family with a novel LGII mutation that was conspicuously absent in the proband with FCD, demonstrating that despite identical clinical symptoms, phenocopies in ADEAF families may exist. This family illustrates that rare epilepsy syndromes within a single family can have both genetic and structural etiologies.

Keywords Epilepsies, partial [C10.228.140.490.360] . Genetic research [C10.228.140.490.360] - LGI1 protein, human [T329500] · Autosomal dominant epilepsy with auditory features - Autosomal dominant partial epilepsy with auditory features - Autosomal dominant lateral temporal lobe epilepsy

5 Core Unit Systems Medicine, University of Wuerzburg, Würzburg, Germany

6 Division Biomedical Genetics, University Medical Center Utrecht, Utrecht, The Netherlands

7 Department of Neurology, Christian-Albrechts-University of Kiel and University Medical Center Schleswig-Holstein, Kiel, Germany

8 Departments of Brain and Cognitive Sciences, Physiology and Cell Biology, Zlotowski Center for Neuroscience, Ben-Gurion University of the Negev, Beer-Sheva, Israel

9 Tel Aviv University Medical School, Ramat Aviv 69978, Israel

10 Division of Neurology, The Children's Hospital of Philadelphia, Philadelphia, PA 19104, USA 


\section{Introduction}

Autosomal dominant (partial) epilepsy with auditory features (ADEAF) is a familial focal epilepsy syndrome with lateral temporal lobe seizure onset in affected family members [1]. Characteristic features are auditory auras and aphasic seizures. Most frequently, simple auditory auras such as humming, buzzing or ringing are reported by affected patients. Complex auditory features such as voices or singing occur less frequently. Affected individuals may also experience other sensory auras including visual as well as motor, psychic or autonomic features [1]. Diagnosis of ADEAF is suspected if $\geq 2$ family members experience auditory auras or aphasic seizures [1].

Mutations in the leucine-rich glioma-inactivated 1 gene (LGI1, Entrez Gene ID 9211, OMIM ID 604619, [1] and, recently, reelin (RELN, Entrez Gene ID 5649, OMIM ID 600514, [2] were reported in about 35 and $17.5 \%$, respectively, of ADEAF families.

Brain imaging is considered unremarkable although in one LGIl-positive family temporal lobe malformations, in particular enlargement of the left temporal lobe, were identified by high-resolution MRI [3]. Furthermore, voxelbased analyses revealed increased anisotropy in the left temporal cortex in a group of LGI1-positive ADEAF patients whose MRI was unremarkable on visual analysis [4].

Here, we report a new ADEAF family including one family member who underwent epilepsy surgery due to right temporal focal cortical dysplasia (FCD). We aim to clarify if FCD can occur in ADEAF, identify the molecular cause and provide a description of the phenotypic range within this family.

\section{Methods}

A large Iranian Jewish family with 14 affected individuals living in Israel was recruited and phenotyped by direct or telephone interviews except for individual II-5 whose history was obtained from his daughter III-9 (Fig. 1). Available medical records were obtained. Routine EEG studies and high-resolution structural 3-T MRI data were collected using a 3-T scanner (Achieva magnet; Philips Healthcare) on the individuals III-12, III-15, III-17 and IV-13 who had consented to these additional studies. A routine epilepsy protocol was used including pre- and post-contrast anatomy scans $(\mathrm{T} 1 \mathrm{~W}, \mathrm{TE} / \mathrm{TR}=3.7 / 8.2 \mathrm{~ms}$, acquisition matrix $432 * 432$, voxel size: $0.5 * 0.5 * 1 \mathrm{~mm}$ ), FLAIR (Axial, TE/ $\mathrm{TR}=125 / 11,000 \mathrm{~ms}, \quad \mathrm{IR}=2800$, acquisition matrix: $272 * 195$, voxel size: $0.5 * 0.5 * 5 \mathrm{~mm})$, DTI (16 directions, voxel size $2 \times 2 \times 2 \mathrm{~mm}$; 50 slices; matrix $116 \times 114$; $\mathrm{TE}=86 \mathrm{~ms}, \quad \mathrm{TR}=6.784 \mathrm{~s}$ ), and T2-weighted (voxel size $0.47 \times 0.59 \times 4 \mathrm{~mm}$; 30 slices; matrix $488 \times 292$; $\mathrm{TE}=90 \mathrm{~ms}, \mathrm{TR}=3 \mathrm{~s}$ ). The slices were orientated perpendicular or parallel to the long axis of the hippocampus.

DNA was extracted from peripheral blood samples. Genotyping was performed using Illumina OmniExpressExome single nucleotide polymorphism genotyping microarrays in 15 family members. Error checking verified the accuracy of the pedigree and indicated high-quality genotyping information. After removing Mendelian and

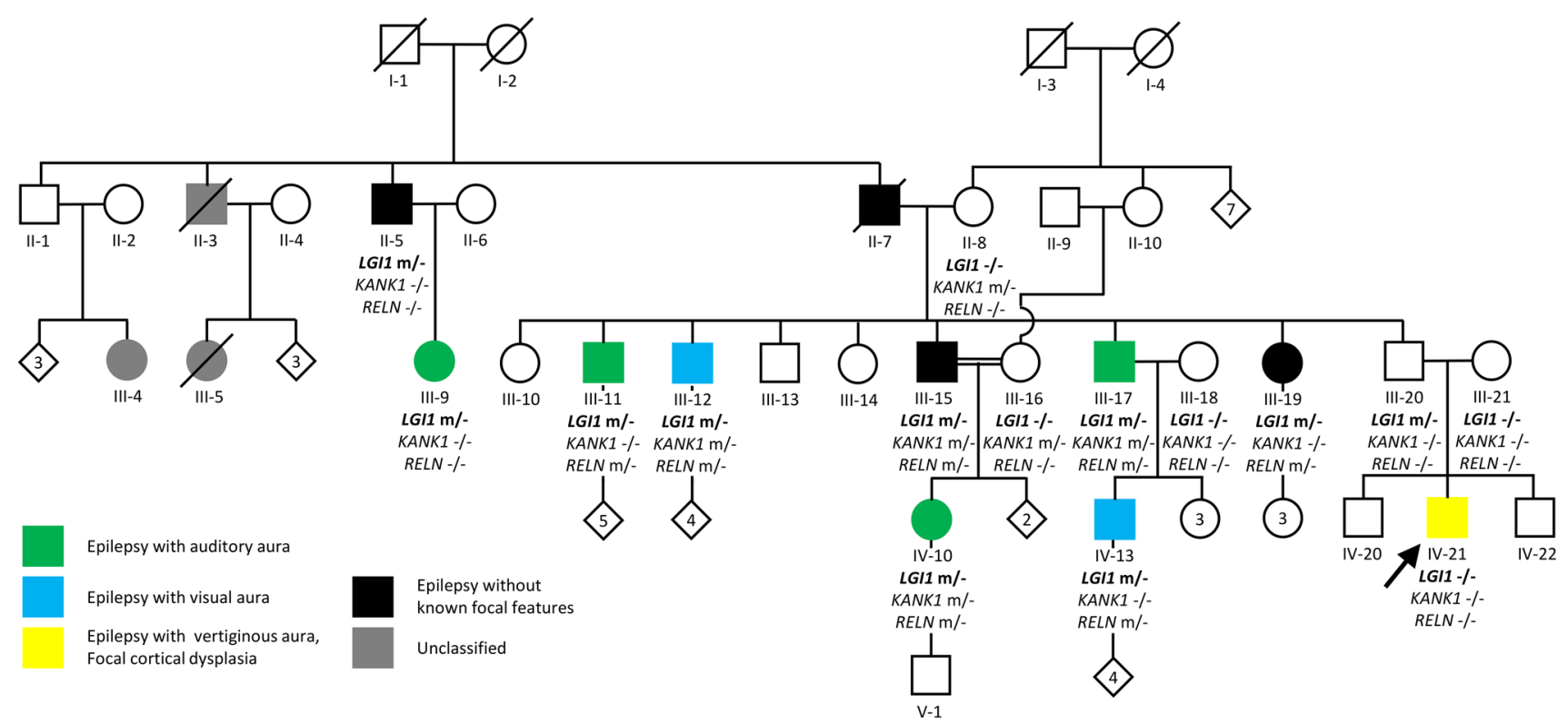

Fig. 1 Pedigree of the family showing segregation of the LGI1 mutation and KANK1 variant. The arrow indicates the proband. LGII leucinerich, glioma-inactivated 1 gene, KANK1 KN motif and ankyrin repeat domains 1 gene, $R E L N$ reelin gene, $m$ mutation/variant present, - wild type 
double recombinant errors, 6312 single nucleotide polymorphism markers were selected for linkage analysis. Exact parametric multipoint linkage analysis was calculated with MERLIN [5]. According to previous reports in ADEAF [6] penetrance was set to 0.7. A phenocopy rate of 0.01 was used reflecting the prevalence of epilepsy in the population. Disease allele frequency was set to 0.0001 as the disease allele was assumed to be rare in the population.

Sanger sequencing of all exons of LGII was performed in individuals III-11 and III-17. Exon 6 was Sanger sequenced in all 15 available family members.

Exome sequencing was performed in individual III-17 using the Illumina HiSeq platform. Segregation analysis of a KN motif and ankyrin repeat domains 1 (KANK1, Entrez Gene ID 23189, OMIM ID 607704) and a RELN variant was performed by Sanger sequencing in all 15 available family members.

\section{Results}

The family included 14 individuals with a history of seizures over three generations. Phenotypic information was available in 11 individuals including the deceased individual II-7 whose history was obtained from his wife and children. Seizure semiology of these 11 individuals was consistent with ADEAF. Median seizure onset was at 15 years. Four family members reported auditory auras, two visual auras and the proband described vertiginous auras (Table 1). Focal dyscognitive seizures with altered awareness or responsiveness occurred in five individuals including two (III-19, IV-13) who reported that they were unable to talk but could understand other people. Individual III-17 reported that he was unable to hear and individuals III-11 and IV-21 had loss of awareness during the focal dyscognitive seizures. Evolution to bilateral convulsive seizures (previously secondarily generalized tonic-clonic seizures) occurred in seven individuals. Three additional family members only had bilateral convulsive seizures without focal features. In four individuals bilateral convulsive seizures occurred exclusively (III-19, IV-19) or predominantly (III-15, IV-13) during sleep. The occurrence of seizures was reported in three further family members but no details were available. Two of these were deceased. Electroencephalographic studies in individuals III-12, III-15, III-17 and IV-13 did not show epileptiform discharges. EEGs done for clinical reasons in individuals III-9, III-19, IV-21 were unremarkable.

Magnetic resonance imaging in the proband IV-21 revealed a right mesiotemporal tumor, which was considered most consistent with a low grade glioma (Fig. 2). The proband underwent surgery and pathology revealed FCD type IIA. Brain MRI in individual III-19 revealed a meningioma in the cerebellopontine angle but no epileptogenic lesion. High-resolution structural 3T-MRIs in individuals III-12, III-15, III-17 and IV-13 were unremarkable.

Epilepsy was mild in most individuals. Five family members became seizure free on medication or had only infrequent seizures when discontinuing medication. Only three individuals continued to have seizures despite taking medication. The proband IV-21 became seizure free after surgery.

Linkage analysis categorizing the proband IV-21 as having unknown affected status revealed peaks (Online Resource 1,2) on chromosome 9p24 (LOD score 2.43) and 10q22-25 (LOD score 2.04). The latter included LGII.

Sanger sequencing of $L G I 1$ revealed a heterozygous mutation in exon 6 (c.641T $>$ C, p.214L $>$ P, NM_005097, Online Resource 3 ) which was predicted to be deleterious by SIFT (score 0) [7] and probably damaging by PolyPhen-2 (score 0.953) [8]. The mutation affects the leucine-rich repeat C-terminal domain by changing an acyclic to a heterocyclic amino acid at a conserved site. The mutation was present in all affected family members except for the proband and has not been reported previously as a pathogenic mutation nor as benign polymorphism in the ExAC database (Exome Aggregation Consortium (ExAC), Cambridge, MA, USA (URL: http://exac.broadinstitute.org) [accessed June 2015]).

Exome sequencing was performed in individual III-17. After filtering for exonic or splice site variants with a frequency $<1 \%$ in the Exome Variant Server (ESP6500_all) and 1000 Genomes (1000 g2012apr, [9] databases and after excluding synonymous variants and segmental duplications, 720 variants remained. None of these variants were located within the linkage region on chromosome 9p24. One variant in exon 9 of KANK1 (c.3296G $>$ C, p. 1099G $>$ A, NM_153186) was located $161 \mathrm{~kb}$ upstream of the linkage region and did not segregate with the phenotype (Fig. 1). Eleven variants in 10 genes were located within the linkage region on chromosome 10q22-25 including the LGII mutation. None of the other 9 genes were considered a likely candidate gene for ADEAF. A variant in exon 45 of RELN (c.7114G $>$ A, p.2372V $>$ M, rs114344654, NM_005045) with a frequency of 22/121246 (0.0001814) in the ExAC database did not segregate with the phenotype (Fig. 1).

\section{Discussion}

Seizure semiology reported by the affected members of this large family was consistent with ADEAF. Four family members reported the typical auditory auras. Visual auras, focal dyscognitive seizures and bilateral convulsive seizures were present as well. The majority of family 


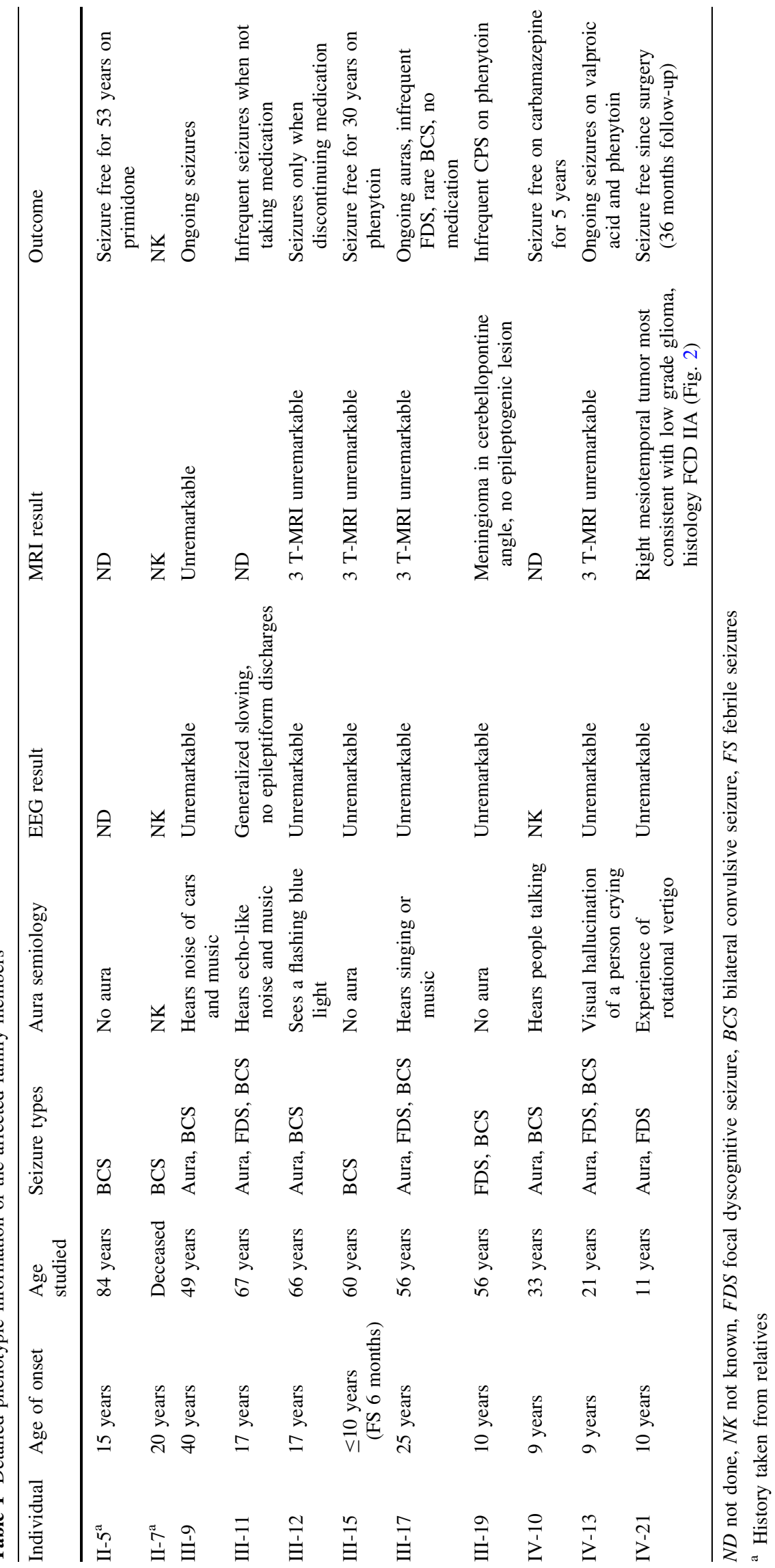


Fig. 2 MRI of the proband showing the right mesiotemporal lesion. a Coronal T2-weighted image, $\mathbf{b}$ axial FLAIR image
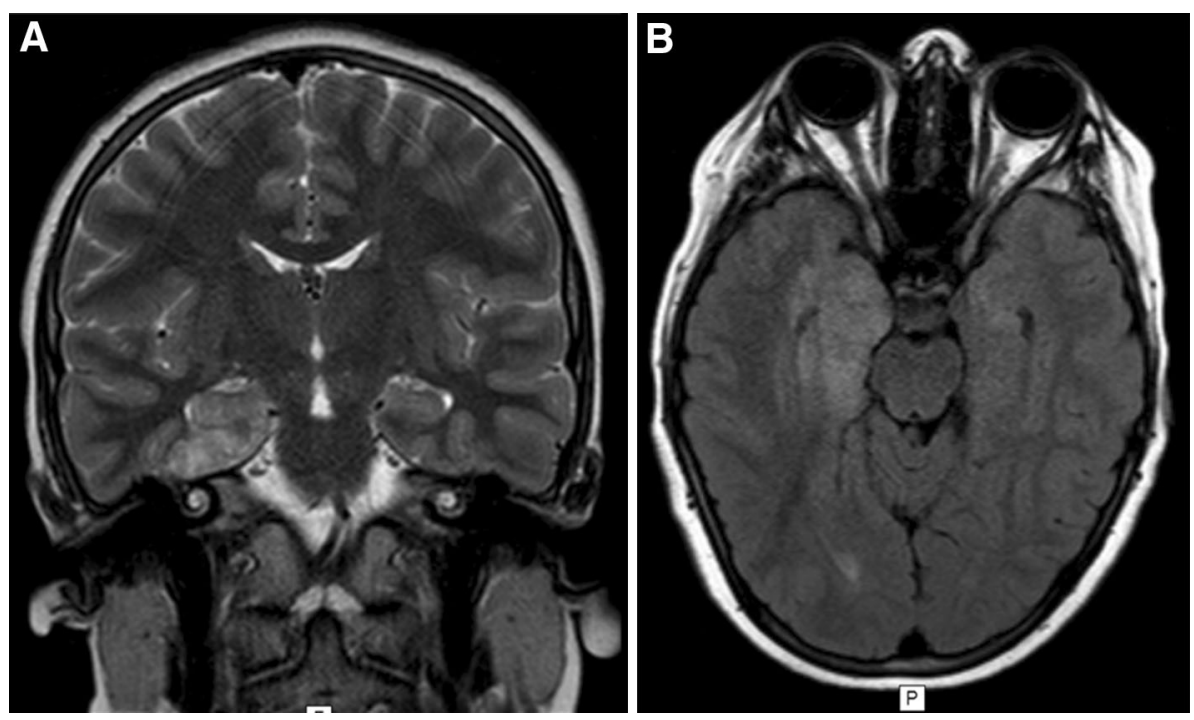

members had infrequent and pharmaco-responsive seizures as is typical for this epilepsy syndrome. EEG studies performed within the framework of our study did not show epileptiform discharges. Although up to two-thirds of individuals with ADEAF may show epileptiform discharges [1] other studies have found similarly low frequencies [10].

The remarkable finding in our family was the presence of right temporal FCD type IIA in the proband. This finding initially raised the question if FCD may be part of the phenotypic spectrum of ADEAF considering two previous reports on temporal lobe abnormalities in ADEAF families $[3,4]$. Furthermore, FCD has recently been reported in familial focal epilepsy with variable foci [11] which is a related focal epilepsy syndrome caused by mutations in DEPDC5 [12]. The proband did not experience auditory auras or aphasic seizures but reported vertiginous auras which also have been described in ADEAF families [13].

However, as most families with ADEAF have not been reported to show MRI abnormalities, the proband was considered to have unknown affected status for the linkage analysis which revealed that he did not carry the chromosome 10 haplotype. Subsequently, sequencing of LGII revealed a novel LGII mutation in the family but not in the proband. The epilepsy phenotype of the proband, therefore, represents a phenocopy as his epilepsy cannot be attributed to the familial genomic LGII mutation. He is likely to have a non-genetic cause of his epilepsy although we cannot exclude the presence of somatic mutations in different genes. Vestibular sensations have been reported during stimulation of the insular, lateral temporal, parietal and frontal cortex $[14,15]$. Propagation of the ictal activity to one of these regions is the likely explanation for the occurrence of a vertiginous aura in the proband resulting in a similar seizure semiology as can be observed in individuals with ADEAF.

In addition to the peak on chromosome 10, which includes the LGIl mutation, linkage analysis revealed another peak on chromosome 9. To clarify whether an identifiable modifier gene may be located within the linkage region on chromosome 9 we performed exome sequencing in one affected individual. No variants were found within the linkage region after filtering but a variant in KANK1 was located very close to the upper boundary. The haplotype at this position was shared by all affected individuals except for III-11. As KANKI had been reported in families with congenital cerebral palsy, hypotonia, quadriplegia and intellectual disability [16, 17], KANKI was considered a plausible candidate modifier gene. However, sequencing of this variant in the other family members revealed that it did not segregate with the phenotype.

Exome sequencing revealed a rare variant in RELN, a gene which has recently been reported in ADEAF [2]. However, incomplete segregation (Fig. 1) and the presence of the variant in controls did not support pathogenicity of this variant. Nevertheless, a modifying effect in the main branch of the family cannot be excluded.

In conclusion, we report a new ADEAF family due to a novel LGII mutation, reinforcing the combination of typical phenotypic features including auditory and visual auras in combination with a usually mild epilepsy, which constitutes the ADEAF spectrum. The proband's phenotype with FCD represents a phenocopy, suggesting that two rare epilepsy syndromes due to both genetic and structural causes can coexist within a single family. Our results do not support that FCD is part of the ADEAF spectrum, in contrast to other familial focal epilepsy syndromes. Given 
that the individual with the phenocopy represented the initial proband for the analysis of the family, our family is an instructive example on how candidate genes for a familial condition may be missed due to phenocopies.

Acknowledgments We thank the family for participation in our study. We also thank the Exome Aggregation Consortium and the groups that provided exome variant data for comparison. A full list of contributing groups can be found at http://exac.broadinstitute.org/ about. The study was supported by the Deutsche Forschungsgemeinschaft with a Trilateral Grant (HE 5415/5-1) and within the EuroEPINOMICS project (HE 5415/3-1), the Christian-AlbrechtsUniversity Kiel and the Philipps-University Marburg.

\section{Compliance with ethical standards}

Ethical standards The study was approved by the local ethics committees of the participating sites and was therefore performed in accordance with the ethical standards laid down in the 1964 Declaration of Helsinki and its later amendments. All participants gave written informed consent prior to inclusion in the study.

Conflicts of interest The authors declare that they have no conflict of interest.

\section{References}

1. Ottman R (1993-2014) Autosomal dominant partial epilepsy with auditory features. In: Pagon RA, Adam MP, Ardinger HH et al (eds) GeneReviews ${ }^{\circledR}$ [Internet]. University of Washington, Seattle

2. Dazzo E, Fanciulli M, Serioli E, Minervini G, Pulitano P, Binelli S, Di Bonaventura C, Luisi C, Pasini E, Striano S, Striano P, Coppola G, Chiavegato A, Radovic S, Spadotto A, Uzzau S, La Neve A, Giallonardo AT, Mecarelli O, Tosatto SC, Ottman R, Michelucci R, Nobile C (2015) Heterozygous reelin mutations cause autosomal-dominant lateral temporal epilepsy. Am J Hum Genet 96(6):992-1000

3. Kobayashi E, Santos NF, Torres FR, Secolin R, Sardinha LA, Lopez-Cendes I, Cendes F (2003) Magnetic resonance imaging abnormalities in familial temporal lobe epilepsy with auditory auras. Arch Neurol 60(11):1546-1551

4. Tessa C, Michelucci R, Nobile C, Giannelli M, Della Nave R, Testoni S, Bianucci D, Tinuper P, Bisulli F, Sofia V, De Feo MR, Giallonardo AT, Tassinari CA, Mascalchi M (2007) Structural anomaly of left lateral temporal lobe in epilepsy due to mutated LGI1. Neurology 69(12):1298-1300
5. Abecasis GR, Cherny SS, Cookson WO, Cardon LR (2002) Merlin-rapid analysis of dense genetic maps using sparse gene flow trees. Nat Genet 30(1):97-101

6. Rosanoff MJ, Ottman R (2008) Penetrance of LGI1 mutations in autosomal dominant partial epilepsy with auditory features. Neurology 71(8):567-571

7. Kumar P, Henikoff S, Ng PC (2009) Predicting the effects of coding non-synonymous variants on protein function using the SIFT algorithm. Nat Protoc 4(7):1073-1081

8. Adzhubei IA, Schmidt S, Peshkin L, Ramensky VE, Gerasimova A, Bork P, Kondrashov AS, Sunyaev SR (2010) A method and server for predicting damaging missense mutations. Nat Methods 7(4):248-249

9. The 1000 Genomes Project Consortium (2012) An integrated map of genetic variation from 1,092 human genomes. Nature 491(7422):56-65

10. Sadleir LG, Agher D, Chabrol E, Elkouby L, Leguern E, Paterson SJ, Harty R, Bellows ST, Berkovic SF, Scheffer IE, Baulac S (2013) Seizure semiology in autosomal dominant epilepsy with auditory features, due to novel LGI1 mutations. Epilepsy Res 107(3):311-317

11. Scheffer IE, Heron SE, Regan BM, Mandelstam S, Crompton DE, Hodgson BL, Licchetta L, Provini F, Bisulli F, Vadlamudi L, Gecz J, Connelly A, Tinuper P, Ricos MG, Berkovic SF, Dibbens LM (2014) Mutations in mTOR regulator DEPDC5 cause focal epilepsy with brain malformations. Ann Neurol 75(5):782-787

12. Dibbens LM, de Vries B, Donatello S, Heron SE, Hodgson BL, Chintawar S, Crompton DE, Hughes JN, Bellows ST, Klein KM, Callenbach PM, Corbett MA, Gardner AE, Kivity S, Iona X, Regan BM, Weller CM, Crimmins D, O'Brien TJ, GuerreroLopez R, Mulley JC, Dubeau F, Licchetta L, Bisulli F, Cossette P, Thomas PQ, Gecz J, Serratosa J, Brouwer OF, Andermann F, Andermann E, van den Maagdenberg AM, Pandolfo M, Berkovic SF, Scheffer IE (2013) Mutations in DEPDC5 cause familial focal epilepsy with variable foci. Nat Genet 45(5):546-551

13. Winawer MR, Ottman R, Hauser WA, Pedley TA (2000) Autosomal dominant partial epilepsy with auditory features: defining the phenotype. Neurology 54(11):2173-2176

14. Mazzola L, Lopez C, Faillenot I, Chouchou F, Mauguiere F, Isnard J (2014) Vestibular responses to direct stimulation of the human insular cortex. Ann Neurol 76(4):609-619

15. Kahane P, Hoffmann D, Minotti L, Berthoz A (2003) Reappraisal of the human vestibular cortex by cortical electrical stimulation study. Ann Neurol 54(5):615-624

16. Lerer I, Sagi M, Meiner V, Cohen T, Zlotogora J, Abeliovich D (2005) Deletion of the ANKRD15 gene at 9p24.3 causes parentof-origin-dependent inheritance of familial cerebral palsy. Hum Mol Genet 14(24):3911-3920

17. Vanzo RJ, Martin MM, Sdano MR, South ST (2013) Familial KANK1 deletion that does not follow expected imprinting pattern. Eur J Med Genet 56(5):256-259 